\title{
The Role of Dopamine in the Nucleus Accumbens and Striatum during Sexual Behavior in the Female Rat
}

\author{
Jill B. Becker, ${ }^{1,2}$ Charles N. Rudick, ${ }^{1}$ and William J. Jenkins ${ }^{1}$ \\ ${ }_{1}^{1}$ Psychology Department, and ${ }^{2}$ Reproductive Sciences Program and Neuroscience Program, The University of Michigan, \\ Ann Arbor, Michigan 48109
}

Dopamine in dialysate from the nucleus accumbens (NAcc) increases during sexual and feeding behavior and after administration of drugs of abuse, even those that do not directly activate dopaminergic systems (e.g., morphine or nicotine). These findings and others have led to hypotheses that propose that dopamine is rewarding, predicts that reinforcement will occur, or attributes incentive salience. Examining increases in dopamine in NAcc or striatum during sexual behavior in female rats provides a unique situation to study these relations. This is because, for the female rat, sexual behavior is associated with an increase in NAcc dopamine and conditioned place preference only under certain testing conditions. This experiment was conducted to determine what factors are important for the increase in dopamine in dialysate from NAcc and striatum during sexual behavior in female rats. The factors considered were the number of contacts by the male, the timing of contacts by the male, or the ability of the female to control contacts by the male. The results indicate that increased NAcc dopamine is dependent on the timing of copulatory stimuli, independent of whether the female rat is actively engaged in regulating this timing. For the striatum, the timing of copulatory behavior influences the magnitude of the increase in dopamine in dialysate, but other factors are also involved. We conclude that increased extracellular dopamine in the NAcc and striatum conveys qualitative or interpretive information about the rewarding value of stimuli. Sexual behavior in the female rat is proposed as a model to determine the role of dopamine in motivated behavior.

Key words: dopamine; microdialysis; nucleus accumbens; striatum; motivation; sexual behavior; incentive salience
The release of dopamine (DA) in the nucleus accumbens (NAcc) and, to a lesser extent, the striatum has been postulated to mediate the reinforcing properties of food, drugs of abuse, and the sexual experience (Wise and Rompre, 1989; Phillips et al., 1991; Robinson and Berridge, 1993). Alternatively, it has been suggested that an increase in extracellular DA in NAcc or striatum is associated with stimuli that predict reinforcement or that this activity attributes incentive salience to the stimuli (Phillips et al., 1993; Schultz et al., 1993; Berridge and Robinson, 1998). By looking at the time when DA increases in the striatum and the NAcc, we can gain additional insight into the roles of these neural structures in motivated behaviors.

Sexual behavior in the female rat is unique among naturally occurring motivated behaviors in that copulation under standard laboratory conditions is not rewarding for the female rat (Oldenburger et al., 1992; Paredes and Alonso, 1997). In female rats and hamsters, there is enhanced DA in dialysate from striatum and NAcc during copulation (Meisel et al., 1993; Mermelstein and Becker, 1995; Pfaus et al., 1995). For female rats, however, this increase in NAcc DA has been found only under conditions in which the female can control or pace the timing of intromissions (Mermelstein and Becker, 1995; Pfaus et al., 1995). Pacing

Received Nov. 2, 2000; revised Jan. 4, 2001; accepted Feb. 8, 2001.

This work was supported by National Science Foundation Grant BNS9816673. W. Jenkins was supported by a fellowship from the National Science Foundation. We thank Kent Berridge and Terry Robinson for helpful comments on an earlier version of this manuscript.

Correspondence should be addressed to Jill B. Becker, Psychology Department, Biopsychology Area, 525 East University, Ann Arbor, MI 48109-1109. E-mail: jbbecker@umich.edu.

Dr. Rudnick's present address: Neuroscience Graduate Program, Northwestern University, Evanston, Illinois 60201.

Copyright $\odot 2001$ Society for Neuroscience $0270-6474 / 01 / 213236-06 \$ 15.00 / 0$ of intromissions determines whether hormones that promote implantation (i.e., the progestational reflex) will be released. When the female rat is pacing, intromissions are spaced $\sim 1-2$ min apart, and the chance that insemination will result in pregnancy is significantly enhanced, compared with reproductive success when the rate of intromissions is at the faster rate of copulation for the male (Adler et al., 1970).

There are individual differences among female rats in the optimal pace of intromissions (Adler, 1978). Each female rat has an individual "vaginal code" that is optimal to induce the progestational reflex in that individual rat (Adler et al., 1970; McClintock and Anisko, 1982; McClintock et al., 1982; McClintock, 1984; Adler and Toner, 1986). In the laboratory situation, pacing behavior occurs if there is a barrier behind which the female rat can escape from the male rat (McClintock, 1984; Erskine, 1989). Furthermore, as mentioned above, the increase in DA concentrations in dialysate from striatum and NAcc of female rats that are pacing copulation is significantly greater than that of female rats that cannot pace or behaviorally receptive animals tested without a male rat (Mermelstein and Becker, 1995). This is true even when pacing females and nonpacing females receive the same number of mounts, intromissions, and ejaculations during an hour of copulatory experience. These results raise the following questions. What is important for the increase in extracellular DA in the NAcc and striatum during pacing of sexual behavior? Is it the amount of copulatory stimuli, the timing of copulatory stimuli, or the act of controlling the copulatory behavior of the male rat that induces the increases in extracellular DA? The results of this experiment will help us to better understand the role of the striatum and NAcc in the sexual experience and in motivated behaviors in general. 


\section{MATERIALS AND METHODS}

Subjects. Adult male and female Long-Evans rats (Charles River Laboratories, Wilmington, MA) weighed 180-200 gm at the beginning of this experiment. Females were housed two or three per cage until they underwent stereotaxic surgery, after which they were housed individually. Male rats were housed in pairs throughout this experiment. All rats were maintained on a $14 / 10 \mathrm{hr}$ light/dark cycle with free access to phytoestrogen-free rat chow (2014 Teklad Global 14\% protein rodent maintenance diet; Harlan, Madison, WI) and water.

Surgical procedures. Female rats were ovariectomized (OVX) by a dorsal approach under methoxyflurane anesthesia $\sim 2$ weeks after arrival. The vaginal epithelium was examined daily by saline lavage for 8 consecutive days after surgery to determine whether all animals were completely OVX.

Stereotaxic surgery was performed under sodium pentobarbital anesthesia (45 mg/kg, i.p.) supplemented with methoxyflurane. Guide cannulas were implanted chronically through the skull and aimed at the dorsolateral striatum and contralateral nucleus accumbens (left-right randomized). The guide cannulas were secured with dental acrylic held on the skull with jeweler's screws. Stereotaxic coordinates (from bregma, skull flat) were as follows: for the dorsolateral striatum, rostral $0.2 \mathrm{~mm}$, lateral $3.2 \mathrm{~mm}$, and ventral $1 \mathrm{~mm}$; for the nucleus accumbens, rostral 1.8 $\mathrm{mm}$, lateral $1.5 \mathrm{~mm}$, and ventral $1 \mathrm{~mm}$.

Behavioral testing. OVX animals that were exhibiting a diestrous vaginal smear continuously were tested for pacing of sexual behavior after subcutaneous priming with $5 \mu \mathrm{g}$ estradiol benzoate (EB) for 3 consecutive days, beginning $72 \mathrm{hr}$ before testing, and with $500 \mu \mathrm{g}$ progesterone $4-6 \mathrm{hr}$ before the behavioral test on the fourth day. The testing chamber $(61 \times 25 \times 46 \mathrm{~cm})$ was Plexiglas, with an opaque wall $(20 \times 0.25 \times 25$ $\mathrm{cm}$ ) that separated the sexual behavior arena (where the male was) from a portion of the chamber in which the female could escape from the male. The female had free access to both sides; the male was trained by passive avoidance to stay on one side. Pacing was defined by the difference in the return latency (time in seconds from contact by male to return of female to the male's side of the arena) between mounts, intromissions, and ejaculations. Females were pacing only if the return latency after mounts was less than the return latency after intromissions, which was less than the return latency after ejaculations. OVX females that did not exhibit a $10 \%$ difference in the intervals between contacts on this test were eliminated from the study ( $n=9$ of 59 rats).

One week after stereotaxic surgery, OVX rats were tested again for pacing behavior as described above. The average return latency after intromissions (for the two pacing sessions) was used as the preferred pacing interval of the animal.

OVX female rats were randomly assigned to one of the following groups (described below): pacing $(n=8)$, preferred pacing interval (PPI; $n=9)$, vaginal mask $(n=8)$, nonpacing $(n=9)$, nonpacing $30 \mathrm{sec}$ interval (NP-30 sec; $n=8$ ), or nonpacing 10 min interval (NP-10 min; $n=8$ ). Before dialysis, all OVX rats were treated with EB and progesterone as described above. The pacing group was tested during dialysis in the pacing chamber. The PPI group was tested in the same chamber with the barrier removed, and the male rat was removed from the chamber after an intromission or ejaculation and returned at the preferred interval of the female $(87-120 \mathrm{sec}$; mean $=100.1 \mathrm{sec})$, as determined in the previous pacing situations. The vaginal mask group was tested under pacing conditions, but with a small piece of masking tape occluding the vagina. The tape was put in place before the initial collection of baseline samples and remained in place throughout dialysis. The nonpacing group was placed in the testing chamber without the opaque barrier, so the male had free access to the female during the time he was in the chamber. The nonpacing interval groups were also tested without the barrier in place, but the male was removed after an intromission or ejaculation and returned either $30 \mathrm{sec}$ or $10 \mathrm{~min}$ later. Behavior was videotaped during the $1 \mathrm{hr}$ period of time that the male was present in the chamber. Behavior was scored by observers blind to the experimental hypothesis. For animals in the pacing chamber, return latency was determined after mounts, intromissions, and ejaculations during each $15 \mathrm{~min}$ interval of dialysis sample collection. For all animals, the number of times the female crossed the center of the chamber (crossings) was determined, as was the number of mounts, intromissions, and ejaculations during each 15 min dialysis sample collection interval.

Microdialysis testing. We used either dialysis probes as described by Robinson and Whishaw (1988) or commercially available probes (CMA/ 11; CMA/Microdialysis AB, Chelmsford, MA). All probes were tested for recovery in vitro at $37^{\circ} \mathrm{C}$ before use as described previously (Becker and Rudick, 1999). Probes that had DA recovery of $18 \pm 4 \%$ for striatum or $12 \pm 4 \%$ for the accumbens were used. The probes were lowered to $6.25 \mathrm{~mm}$ (4 mm dialysis membrane) for striatum or $8.25 \mathrm{~mm}(2 \mathrm{~mm}$ dialysis membrane) for accumbens. Microdialysis probes were inserted into the dorsolateral striatum and contralateral nucleus accumbens under methoxyflurane anesthesia $12-18 \mathrm{hr}$ before the collection of samples. The flow rate through the probe was $1.5 \mu \mathrm{l} / \mathrm{min}$, and samples were collected at $15 \mathrm{~min}$ intervals. The concentrations of DA, dihydroxyphenylacetic acid (DOPAC), and homovanillic acid (HVA) were determined in dialysate using HPLC and electrochemical detection as described previously (Becker and Rudick, 1999). The average of two baseline samples was used to determine the basal extracellular concentration of DA, DOPAC, and HVA (corrected for percentage of recovery). All values are expressed as femtomol in $15 \mu \mathrm{l}$ of dialysate.

Histology. At the conclusion of microdialysis, females were given a lethal injection of sodium pentobarbital and intracardial perfusions of $0.9 \%$ saline followed by $4 \%$ formalin. The position of the probe in the dorsolateral striatum or NAcc was determined from cresyl violet-stained $50 \mu \mathrm{m}$ sections. The site of the lesion was determined by an observer blind to the treatment of the animal. Dialysis data were excluded for four animals with dialysis probes in the striatum and eight animals with probes in the NAcc, but these were not the same animals and were distributed across the groups. Dialysis probes in the NAcc were predominantly found to be in the core $(n=34)$, but a few were in the core-shell boundary $(n=4)$ or solely in the shell $(n=4)$. The variation in core-shell distribution was randomized across groups, and dialysis data did not vary with probe placement. Final numbers for dialysate data of each group are indicated in the legends to the Figures. Behavior was analyzed for all animals.

Statistical analyses. Data were evaluated with repeated measures ANOVA to determine whether there were group differences. Post hoc comparisons at individual time points were made using the BonferroniDunn correction. Comparisons within a group to determine whether there had been a change in extracellular DA during exposure to the male were done with paired $t$ tests. All analyses were conducted using Statview $4.5+$ for the Macintosh computer.

\section{RESULTS}

\section{Nucleus accumbens}

DA in dialysate from NAcc increased significantly more than baseline during the hour that the male rat was present in the testing chamber for all groups except for the NP-10 min group (mean during baseline vs mean during hour with male present; paired $t$ tests; $p<0.05$ ). The increase in extracellular DA was significantly greater for the pacing and PPI groups than for all other groups (Fig. 1) (main effect of group, $F_{(5,42)}=9.49 ; p<$ $0.0001)$. On pairwise comparisons, the increases in extracellular DA for the pacing and PPI groups were significantly greater than for all other groups $(p<0.003)$, and there were no other differences between groups. The increases in extracellular DA for the pacing and PPI groups did not differ from each other.

As can be seen in Figure 1, there were small differences in the basal extracellular DA, with the NP-10 min group starting the experiment with higher basal DA in dialysate than the nonpacing, vaginal mask, or NP-30 sec groups. The NP-30 sec group began with lower extracellular DA than the vaginal mask, pacing, and PPI groups. Basal extracellular DA concentrations had no apparent influence on whether an animal showed an increase in extracellular DA during the testing period.

\section{Striatum}

DA in dialysate from striatum increased significantly from baseline during the hour that the male rat was present in the testing chamber for all groups except for the NP-10 min and NP-30 sec groups (mean during baseline vs mean during hour with male present; paired $t$ tests; $p<0.02$ ). The increase in extracellular DA was significantly greater for the pacing group and the PPI group than for all other groups (Fig. 2) (main effect of group, $F_{(5,40)}=$ 


\section{DA IN DIALYSATE - NACC}

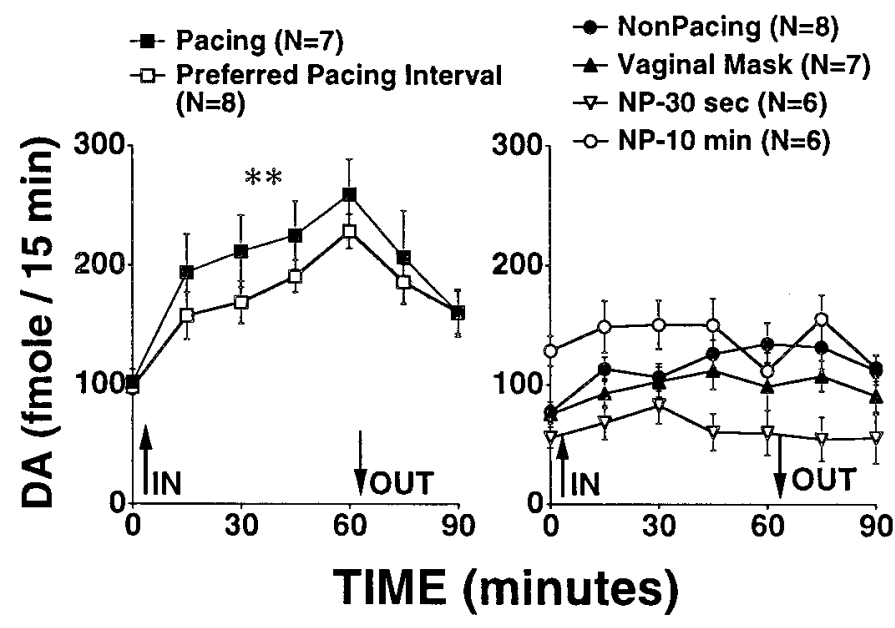

Figure 1. DA concentrations in dialysate $($ mole $/ 15 \mathrm{~min})$ obtained from nucleus accumbens of sexually receptive female rats. The value obtained for time 0 is the mean of two 15 min baseline samples obtained immediately before the introduction of the male rat into the chamber. Values indicate the mean \pm SEM. ${ }^{*}$ The increase in DA in dialysate during the time the male was present was significantly greater for the pacing and PPI groups than for all other groups $(p<0.003)$. There were no other differences among the groups.

\section{DA IN DIALYSATE - STRIATUM}

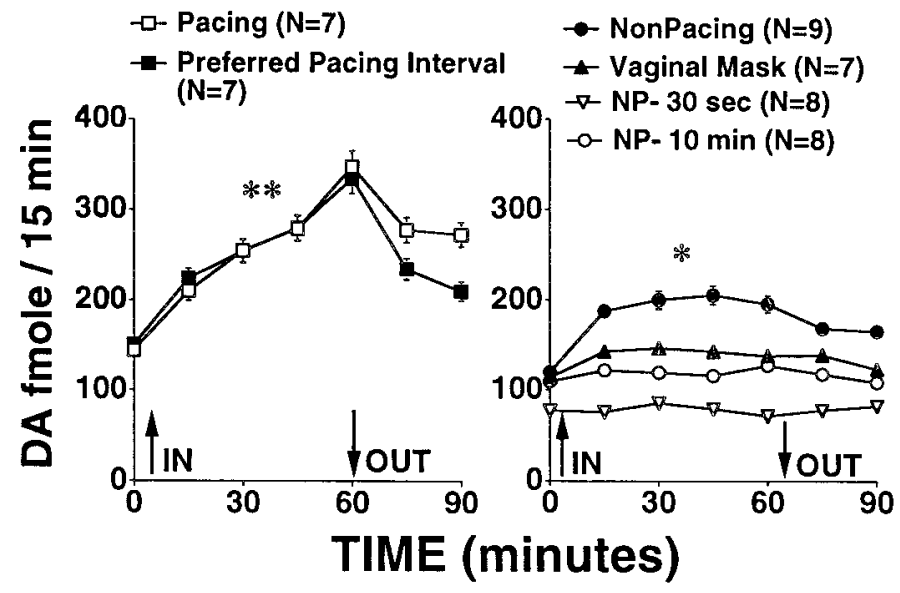

Figure 2. DA concentrations in dialysate ( $\mathrm{fmole} / 15 \mathrm{~min}$ ) obtained from striatum of sexually receptive female rats. Values indicate the mean \pm SEM. ${ }^{* *}$ The increase in DA in dialysate during the time the male was present was significantly greater for the pacing and PPI groups than for all other groups $(p<0.003)$. *The increase in DA in dialysate during the time the male was present was significantly greater for the nonpacing group than for the NP-10 min and NP-30 sec groups $(p<0.0033)$.

16.68; $p<0.0001)$. On pairwise comparisons, the increase in extracellular DA for the pacing and PPI groups was significantly greater than for all other groups $(p<0.003)$ and did not differ from one to the other. The increase in extracellular DA for the nonpacing group was significantly greater than that seen in the NP-10 min and NP-30 sec groups $(p<0.0033)$.

As can be seen in Figure 2, there were also small differences in the basal extracellular DA, with the NP-30 sec group beginning the experiment with lower extracellular DA than the pacing, PPI, and nonpacing groups. Basal extracellular DA concentrations had no apparent influence on whether an animal showed an increase in extracellular DA during the testing period.

The amount of HVA and DOPAC detected in dialysate from both NAcc and striatum increased during the period of time when the male rat was in the chamber, but there were no differences among groups in either brain region (data not shown).

\section{Behavior}

As can be seen in Figure $3 A$, the vaginal mask group received the largest number of mounts during the first 15 min interval. Over the entire hour, the NP-10 min group received fewer mounts than the vaginal mask group and the NP-30 sec group (Fig. $3 A)(p<$ $0.005)$. This is most likely an artifact of the male being repeatedly removed from the chamber for $10 \mathrm{~min}$ in the NP-10 min group.

When the number of intromissions received by the pacing, nonpacing, NP-30 sec, and PPI groups were compared, there was a significant effect of group (Fig. $3 A)\left(F_{(3,30)}=4.986 ; p=0.0063\right.$; the vaginal mask group did not receive intromissions, the NP-10 min group received very few intromissions, and both were excluded to avoid skewing the statistics). In pairwise comparisons, the nonpacing group received more intromissions plus ejaculations than did the pacing or PPI groups $(p<0.01)$, and the NP-30 sec group received more intromissions plus ejaculations than did the PPI group $(p<0.01)$.

Finally, all rats were active during the hour that the male rat was present in the testing chamber, with all animals exhibiting at least 25 crossings across a midline in the chamber. The nonpacing group exhibited more crossings than the NP-10 min, the PPI, the vaginal mask, or the pacing groups $(p<0.0033)$. The NP-30 sec group exhibited more crossings than the NP-10 min group and the pacing group $(p<0.0033)$.

When looking at the behavior engaged in by the female, the latency after a contact until the next male-female contact occurs can be examined to determine the temporal pattern of coital stimulation that the female receives. As seen in Figure 4, females in the pacing group had the longest intervals after ejaculations, with periods that were significantly longer than the nonpacing or PPI groups $(p<0.008)$. The pacing and PPI groups had longer periods after intromissions than did the other groups $(p<0.008)$. Finally, the PPI group had shorter latencies after mounts than the NP-30 sec group $(p<0.008)$.

\section{DISCUSSION}

The results of this experiment indicate that the timing of copulatory stimuli is critical for the magnitude of the increase in extracellular DA in dialysate from the NAcc. Although nonpacing and NP-30 sec animals received the greatest number of intromissions and ejaculations, the pacing and PPI groups had the greatest increase in DA in dialysate from the NAcc. For the striatum, copulatory stimuli that occurred at the preferred interval of the female also induced the greatest increase in extracellular DA. This was true whether or not the female actively controlled the rate of the interval. The increase in striatal DA seen for the nonpacing group, however, was greater than for groups in which the male had been removed and returned at intervals other than that preferred by the female. The data from the PPI group indicate that the female rat does not need to be actively engaged in behaviors associated with pacing (i.e., leaving the male or returning to the male) in order for the increase in extracellular DA in NAcc or striatum to be greater than that seen under all other testing conditions. The significant increase in extracellular DA in the PPI group, in contrast with the lack of increase in 

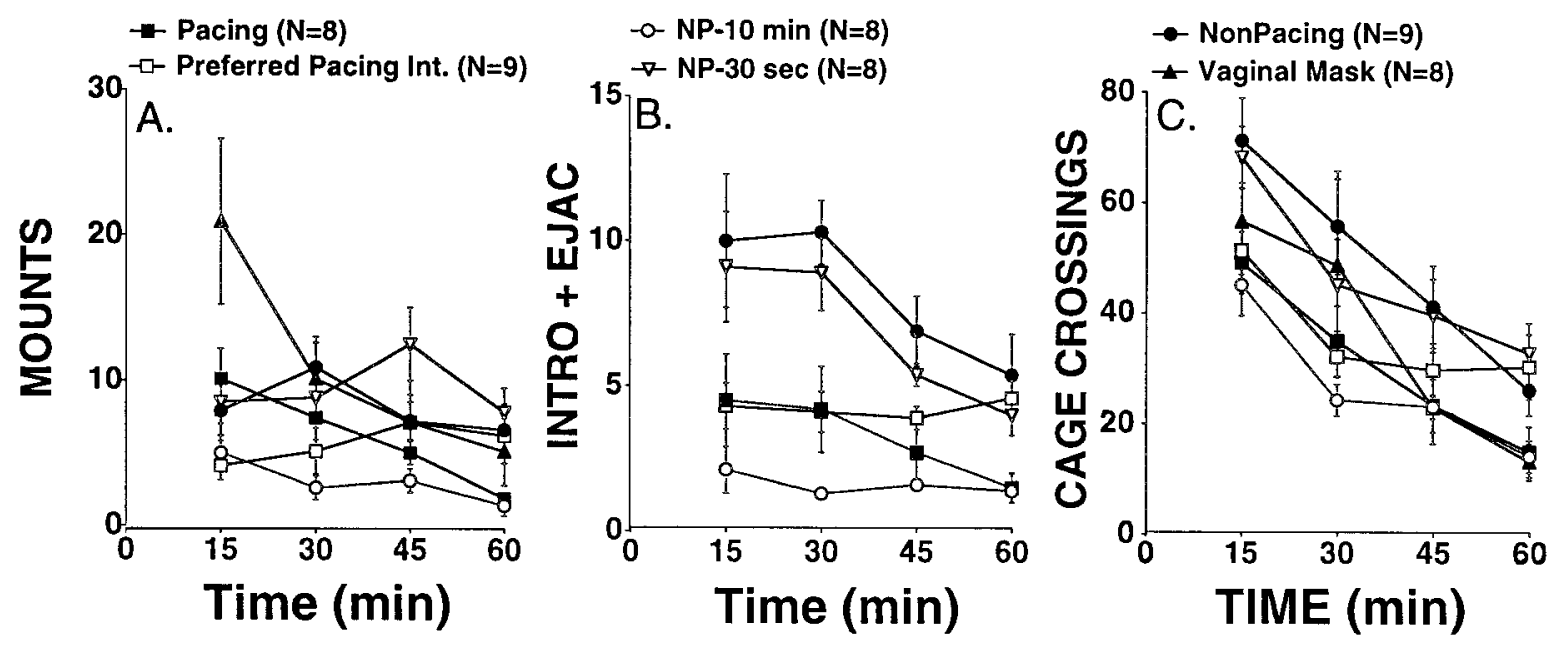

Figure 3. Sexual behavior $(A, B)$ and activity $(C)$ during the hour that the male was in the testing chamber with the female rat. $A$, Mounts received by females during each of the 15 min sample collection periods when the male was present. $B$, Intromissions plus ejaculations received by females during each of the 15 min sample collection periods when the male was present. The nonpacing group received more intromissions plus ejaculations than did the pacing or PPI groups $(p<0.01)$. The NP-30 sec group received more intromissions plus ejaculations than did the PPI group $(p<0.01)$. $C$, General activity (number of times crossing a midline in the cage) during the hour that the male rat was present in the chamber with the female rat. Collection periods were times when the male was present. The nonpacing group made more cage crossings than did the NP-10 min, vaginal mask, pacing, or PPI groups $(p<0.003)$. The NP-30 sec group made more cage crossings than did the NP-10 min or pacing groups $(p<0.0033)$.

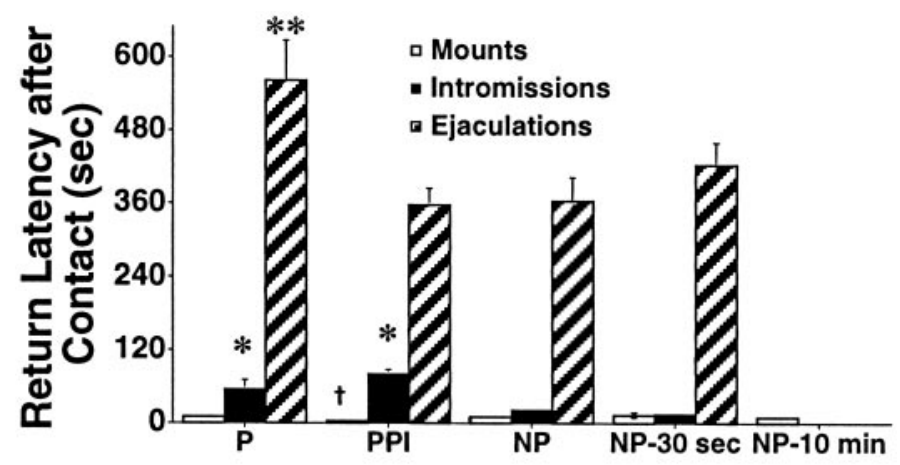

GROUP

Figure 4. Latency after a contact with the male rat before the next male-female contact for each group. Data not shown for the NP-10 min group because the values were artificially controlled by the experimenter and were always $>10 \mathrm{~min}$. These data were also not included in the data analyses for return latency for the same reason. Histograms indicate the mean; error bars indicate \pm SEM. $P$, Pacing; PPI, preferred pacing interval; $N P$, nonpacing; $N P-30 \mathrm{sec}$, nonpacing-30 sec group; $N P-10 \mathrm{~min}$, nonpacing 10 min group. *The pacing and PPI groups had longer periods after intromissions than did the other groups $(p<0.008)$. **The interval after an ejaculation was longer for animals in the pacing group than the nonpacing or PPI groups $(p<0.008)$. ${ }^{\dagger}$ The PPI group had shorter periods after mounts than did the NP-30 sec group $(p<0.008)$.

extracellular DA in groups with shorter (NP-30 sec) or longer (NP-10 min) inter-intromission intervals, supports the idea that the timing of the coital stimulation is critical for the DA increase. The results from the vaginal mask group indicate that the pacing apparatus and the presence of a male rat can induce a small increase in extracellular DA in NAcc or striatum, but in the absence of vaginocervical stimulation this increase is significantly lower than for the pacing or PPI groups.

One could postulate that the increase in DA in dialysate from the NAcc and striatum is dependent on the quantity of intromissions and ejaculations. If this were the case, then one would have expected an initial DA increase in the nonpacing and NP-30 sec groups. These two groups received $\sim 20$ intromissions plus ejaculations per $15 \mathrm{~min}$, whereas the pacing and PPI groups received less than five intromissions plus ejaculations per 15 min (Fig. 3B). In the NAcc, there was a small increase in extracellular DA during the hour that the male rat was in the chamber for the nonpacing and NP-30 sec groups. For the striatum, the DA increase was significantly greater for the nonpacing group than the NP-30 sec and NP-10 min groups. During all intervals, however, the increase in NAcc and striatal DA for the pacing and PPI groups was significantly greater than all other groups. Thus, the DA response is not a measure of how much vaginocervical stimulation has been received. The increase in DA in the striatum and NAcc is also not related to locomotor activity because the nonpacing and NP-30 sec groups were also more active than the other groups, and yet had lower extracellular DA.

Pacing behavior in female rats has only recently become a topic of laboratory investigation (Erskine, 1989). Sexual behavior in the female rat has typically been studied in the laboratory under conditions in which the male rat is able to copulate with the female rat at will. This results in low levels of female-initiated contacts and high rates of reflexive and defensive behaviors in the female rat. Using seminatural conditions, it was observed that the female rat actively controls the pace of copulatory behavior by exhibiting hopping and darting behaviors as well as by actively withdrawing from the male (McClintock, 1984). The evolutionary importance of pacing behavior for reproductive success is evident. For the male rat, a rapidly paced series of intromissions $(<1$ min between intromissions) is optimal to induce ejaculation in the fewest number of intromissions (Adler, 1978). The female rat, on the other hand, requires behavioral activation of a progestational reflex. When intromissions are paced by the female, the chance that insemination will result in pregnancy is enhanced significantly (Adler, 1978). These sexually dimorphic mating strategies are optimal for the reproductive success of both males and females. In the wild, mating is reported to occur within a group of animals, rather than in individual male-female pairs. 
With rapid intromissions and ejaculation, the mating strategy of the male maximizes the number of females he is able to inseminate. The pacing behavior of the female increases the probability that pregnancy will occur.

In addition to enhanced fertility with pacing behavior, the female rat develops a preference for a place in which she has engaged in sex if she can pace the rate of intromissions (Oldenburger et al., 1992; Paredes and Alonso, 1997). On the other hand, female rats do not develop a preference for a place in which they engaged in sex under standard laboratory conditions (Oldenburger et al., 1992). Thus, engaging in sexual behavior when pacing of intromissions is possible is associated with increased DA in striatum and NAcc and is reinforcing for the female rat.

In a recent study from this laboratory, we found that female rats with bilateral NAcc lesions that include the shell are more likely to avoid sexual contact with a male than are animals with control lesions or lesions of the NAcc core (Jenkins and Becker, 2001). These results suggest that sexual motivation is mediated by the NAcc, in particular the shell portion of the NAcc. In the present study, the location of the probes within the NAcc was examined post hoc. Most probes were placed within the core of the NAcc. Results of selective microdialysis in shell versus core of the NAcc suggest that increases in DA would be even greater, but in the same direction, if probes had been placed in the shell selectively (Sokolowski et al., 1998.). There are not enough data from this experiment, however, to address this issue.

The results from this experiment indicate that the increase in DA in dialysate from the NAcc is not a passive response to coital stimulation or copulation-related motor activity. Instead, it reflects qualitative information about the timing of copulatory stimuli received. In the striatum, however, an increase in DA can also be induced by coital stimulation not received at the preferred interval of the female, as seen in the nonpacing group. Thus, the timing of coital stimulation does not appear to be as critical for the increase in DA in striatum as it is for the DA increase in the NAcc.

Conditioned place preferences are formed when female rats are pacing sexual behavior, but not when they engage in nonpaced sex (Oldenburger et al., 1992). From these studies, we infer that paced sexual behavior is rewarding. Taken with the findings of this experiment, results suggest that the increase in NAcc DA in the PPI group indicates that the copulatory stimuli have been interpreted as being rewarding. Experiments in progress will test the hypothesis that introducing the male at the PPI of the female is sufficient to induce a preferred place preference.

The question can then be raised about whether the increase in NAcc DA during paced copulation indicates the hedonic value of the stimulation or its incentive salience (i.e., liking vs wanting). If the increase in NAcc DA reflects the hedonic value of stimuli, then during nonpaced sexual behavior the female could experience pleasure with the first few initial intromissions, coincident with an increase in NAcc DA. However, when intromissions occur too frequently (or too infrequently), the sensation would lose hedonic value, and DA would decrease. During paced sexual behavior, if samples are obtained at appropriate intervals, shorter than those in this experiment, DA should rise during an intromission and fall before the female reinitiates contact with the male to seek another intromission. A comparable pattern is seen during self-administration of cocaine (Wise et al., 1995). On the other hand, if NAcc DA attributes incentive salience to the sexual experience, one would predict that the DA increase would not occur in the PPI group until after a few intromissions have been received at the preferred interval. Furthermore, if NAcc DA attributes incentive salience, DA should increase as the female reinitiates contact with the male. In other words, because it is the timing of the stimuli that is either wanted or liked, the timing of the increase in NAcc DA, relative to when a female receives an intromission, can be used to learn what role DA is playing in this regard.

The finding that the magnitude of the increase in NAcc DA did not differ between groups that were actively pacing sexual behavior and those in the PPI group suggests that this neural system is not specifically mediating the control or initiation of behaviors to seek reinforcement. The converse is true. This DA system is activated as a consequence of the copulation occurring at the preferred interval of the female. These data also suggest that the DA system is not primarily interested in signals which predict that a reward will occur. Taken with the finding that NAcc lesions that include the shell inhibit the initiation of sexual behavior in female rats (Jenkins and Becker, 2001), it is possible that information from DA in the NAcc is interpreted by intrinsic neurons to induce the female to seek the male (in this instance).

We conclude that the role of DA in the NAcc and, to a lesser extent, the striatum is to convey qualitative or interpretive information about the rewarding value of stimuli. Because of the unique properties of sexual behavior in the female rat, we maintain that this system is uniquely designed to be able to determine whether the value attributed is caused by the hedonic value of the stimuli or its incentive salience.

\section{REFERENCES}

Adler NT (1978) On the mechanisms of sexual behavior and their evolutionary constraints. In: Biological determinants of sexual behavior (Hutchison JB, ed), pp 657-694. New York: Wiley.

Adler NT, Toner JPJ (1986) The effects of copulatory behavior on sperm transport and fertility in rats. Ann NY Acad Sci 474:21-32.

Adler NT, Resko JA, Goy RW (1970) The effect of copulatory behavior on hormonal change in the female rat prior to implantation. Physiol Behav 5:1003-1007.

Becker JB, Rudick CN (1999) Rapid effects of estrogen or progesterone on the amphetamine-induced increase in striatal dopamine are enhanced by estrogen priming: a microdialysis study. Pharmacol Biochem Behav 64:53-57.

Berridge KC, Robinson TE (1998) The role of dopamine in reward: hedonics, learning and incentive salience after 6-hyproxydopamine lesions. Brain Res Rev 28:309-369.

Erskine MS (1989) Solicitation behavior in the estrous female rat: a review. Horm Behav 23:473-502.

Jenkins WJ, Becker JB (2001) Role of the striatum and nucleus accumbens in paced copulatory behavior in the female rat. Behav Brain Res, in press.

McClintock MK (1984) Group mating in the domestic rat as context for sexual selection: consequences for the analysis of sexual behavior and neuroendocrine responses. Adv Study Behav 14:1-50.

McClintock MK, Anisko JJ (1982) Group mating among Norway rats. I. Sex differences in the pattern and neuroendocrine consequences of copulation. Anim Behav 30:398-409.

McClintock MK, Anisko JJ, Adler NT (1982) Group mating among Norway rats. II. The social dynamics of copulation: competition, cooperation, and mate choice. Anim Behav 30:410-425.

Meisel RL, Camp DM, Robinson TE (1993) A microdialysis study of ventral striatal dopamine during sexual behavior in female Syrian hamsters. Behav Brain Res 55:151-157.

Mermelstein PG, Becker JB (1995) Increased extracellular dopamine in the nucleus accumbens and striatum of the female rat during paced copulatory behavior. Behav Neurosci 109:354-365.

Oldenburger WP, Everitt BJ, de Jonge FH (1992) Conditioned place preference induced by sexual interaction in female rats. Horm Behav 26:214-228.

Paredes RG, Alonso A (1997) Sexual behavior regulated (paced) by the female induces conditioned place preference. Behav Neurosci 111:123-128.

Pfaus JG, Damsma G, Wenkstern D, Fibiger HC (1995) Sexual activity increases dopamine transmission in the nucleus accumbens and striatum of female rats. Brain Res 693:21-30.

Phillips AG, Pfaus JG, Blaha CD (1991) Dopamine and motivated be- 
havior: insights provided by in vivo analyses. In: The mesolimbic dopamine system: from motivation to action (Willmer P, Scheel-Kruger J, eds), pp 199-224. New York: Wiley.

Phillips AG, Atkinson LJ, Blackburn JR, Blaha CD (1993) Increased extracellular dopamine in the nucleus accumbens of the rat elicited by a conditioned stimulus for food: an electrochemical study. Can J Physiol Pharmacol 71:387-393.

Robinson TE, Berridge KC (1993) The neural basis of drug craving: an incentive-sensitization theory of addiction. Brain Res Rev 18:247-291.

Robinson TE, Whishaw IQ (1988) Normalization of extracellular dopamine in striatum following recovery from a partial unilateral 6-OHDA lesion of the substantia nigra: a microdialysis study in freely moving rat. Brain Res 450:209-224.
Schultz W, Apicella P, Ljungberg T, Romo R, Scarnati E (1993) Rewardrelated activity in the monkey striatum and substantia nigra. Prog Brain Res 99:227-235.

Sokolowski J, Conlan A, Salamone J (1998) A microdialysis study of nucleus accumbens core and shell dopamine during operant responding in the rat. Neuroscience 86:1001-1009.

Wise RA, Rompre P-P (1989) Brain dopamine and reward. Annu Rev Psychol 40:191-225.

Wise RA, Newton P, Leeb K, Burnette B, Pocock D, Justice JBJ (1995) Fluctuations in nucleus accumbens dopamine concentration during intravenous cocaine self-administration in rats. Psychopharmacology (Berl) 120:10-20. 